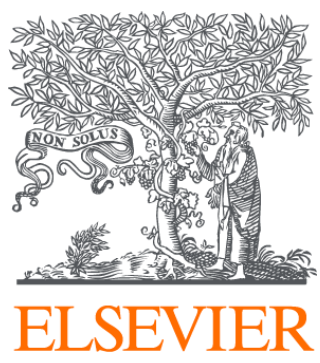

Since January 2020 Elsevier has created a COVID-19 resource centre with free information in English and Mandarin on the novel coronavirus COVID-

19. The COVID-19 resource centre is hosted on Elsevier Connect, the company's public news and information website.

Elsevier hereby grants permission to make all its COVID-19-related research that is available on the COVID-19 resource centre - including this research content - immediately available in PubMed Central and other publicly funded repositories, such as the WHO COVID database with rights for unrestricted research re-use and analyses in any form or by any means with acknowledgement of the original source. These permissions are granted for free by Elsevier for as long as the COVID-19 resource centre remains active. 
with disease severity and severe acute respiratory syndrome coronavirus 2 (SARS-CoV-2) infectivity, highlighting a potential beneficial role of commensal bacteria in combating SARS-CoV-2 infection. Analysis of blood samples showed that the microbial imbalance found in the COVID patients was also associated with raised levels of inflammatory cytokines and blood markers of tissue damage. ${ }^{3}$ These data suggest that the gut microbiome might influence the immune system response to COVID-19 infection and potentially affect disease severity and clinical outcome.

As mentioned in Marazzato et al's letter, probiotics could suppress gut pathogens by competing for nutrients or secretion of certain metabolites. Moreover, they might also constitute part of an "ecogroup" that maintain consistent covariation with other beneficial commensals. ${ }^{4}$ This could be achieved by modulation of host immunity or by a cross-feeding effect between probiotics Bifidobacteria with butyrate-producing colonic bacteria, such as Faecalibacterium prausnitzii via the production of acetate. ${ }^{5}$ Therefore, oral bacteriotherapy not only replenish the beneficial bacteria per se, but may also favor the coexistence of other commensals that were depleted in COVID-19 patients.

Alterations of both bacterial and fungal microbiome in a subset of COVID-19 patients persisted even after clearance of SARS-CoV-2 virus from nasopharyngeal samples and resolution of respiratory symptoms. ${ }^{2,3}$ This underscores a potential prolonged detrimental effect of secondary infection on host health after SARS-CoV-2 infection cautioning on monitoring of patients with COVID-19 even after recovery. Although coronavirus passes quickly for most, some people are experiencing long-term effects, including persistent symptoms of fatigue, dyspnea, and joint pains, that continued for weeks or months after initial onset of symptoms. In this regard, we posit that the dysbiotic gut microbiome could contribute to immune-related health problems post COVID-19 and future long-term observational studies are needed to confirm this hypothesis.

Although some probiotic formulation has been used in small case series in COVID-19 with initial promising results, not all probiotics are the same. ${ }^{6}$ We agree that blind use of conventional probiotics for COVID-19 is not recommended. Robust scientific and clinical data in COVID-19 patients will be required to support the use of existing or new formulations. It is likely that a more targeted approach to modulation of gut microbiota as one of the therapeutic approaches of COVID-19 and its comorbidities will be necessary. The Chinese University of Hong Kong team recently developed an oral microbiome immunity formula for COVID-19 patients and postulated that this formula would improve clinical symptoms and decrease proinflammatory markers in SARS-CoV-2 infection. In a pilot study in patients hospitalized with COVID-19 (unpublished work, Clinical Trials Registry NCT04581018), significantly more patients who received the formula achieved symptom resolution and a reduction in pro-inflammatory immune markers than those who had standard care. Relative abundance of favorable bacteria species also significantly increased after synbiotic therapy compared with baseline. ${ }^{3}$
These preliminary data suggest that modulation of gut microbiota offers hope of boosting immunity against COVID19 and other emerging viral infections. Whether improvement of clinical symptoms after probiotics supplementation was partly contributed by suppression of fungal pathogens such as Candida spp warrants further investigation. We believe that a formulation targeting certain microbes that were depleted in COVID-19 patients would achieve the most benefit for patients and delineating mechanisms of oral bacteriotherapy with anti-SARS-CoV-2 functions present opportunities for discovery of novel microbial therapeutics to prevent and treat COVID-19. ${ }^{7}$

\section{ZHILU XU}

FRANCIS K.L. CHAN

SIEW C. NG

Center for Gut Microbiota Research

The Chinese University of Hong Kong

Hong Kong, China and

Department of Medicine and Therapeutics

LKS Institute of Health Science

The Chinese University of Hong Kong

Hong Kong, China

\section{References}

1. Chiu L, et al. Front Immunol 2017;8:1678.

2. Zuo T, et al. Gastroenterology 2020;159:1302-1310.e5.

3. Yeoh YK, et al. Gut 2021;70:698-706.

4. Raman AS, et al. Science 2019;365:eaau4735.

5. Rivière A, et al. Front Microbiol 2016;7:979.

6. Mak JWY, et al. Lancet Gastroenterol Hepatol 2020; 5:644-645.

7. Hu J, et al. Trends Food Sci Technol 2021;108:187-196.

\section{Conflicts of interest}

The authors disclose no conflicts.

Most current article

https://doi.org/10.1053/j.gastro.2021.01.198

\section{Impact of COVID-19 Outbreak on the Management of Patients With Severe IBD: A Domino Effect}

Dear Editors:

The International Organization for the Study of Inflammatory Bowel Diseases recently published a consensus ${ }^{1}$ in Gastroenterology about the management of patients with inflammatory bowel disease (IBD) during the coronavirus disease 2019 (COVID-19) pandemic, addressing several topics of interest, such as the risk of infection in IBD patients, how to manage therapies, and how to safely provide continuity of biologic therapy. We read it with great interest and we highly 
appreciated the effort to provide guidance to IBD care in these difficult days, even in the absence of evidence-based data.

Indeed, as IBD physicians working in one of the most severely affected regions of the world (Lombardia region, in northern Italy ${ }^{2}$ ), we had to face additional and unexpected difficulties while managing severe IBD during the SARSCoV-2 (severe acute respiratory syndrome coronavirus 2) outbreak.

Here, we report the emblematic case of a 38-year-old man. Because of episodes of bloody diarrhea, the patient underwent colonoscopy in January 2020 with evidence of mild proctosigmoiditis, with histology compatible with ulcerative colitis. A gastroenterology consult was scheduled for the end of February, but not performed due to the COVID-related limitations to nonurgent consultations and procedures. A short course of oral mesalamine therapy given by the general practitioner provided clinical remission, but no maintenance therapy was initiated. After 2 months, the patient developed severe bloody diarrhea ( $>10$ episodes/d), malaise, and diffuse abdominal pain. For these symptoms, he called the emergency service twice, who telephonically suggested to avoid access to the hospitals because of the COVID-19 outbreak. After 2 weeks at home his clinical conditions further deteriorated and he was finally transported to the emergency department of our hospital (Policlinico San Donato, a University Hospital in the Southeastern region of Milan metropolitan area). He appeared severely ill, tachycardic, with mild fever $\left(37.8^{\circ} \mathrm{C}\right)$ and diffuse abdominal pain. Laboratory tests showed markedly elevated Creactive protein $(24 \mathrm{mg} / \mathrm{dL}$, normal values $<0.5 \mathrm{mg} / \mathrm{dL})$, neutrophilic leukocytosis and hypoalbuminemia $(2.7 \mathrm{~g} / \mathrm{dL})$. Chest x-ray was normal and nasopharyngeal swab for novel coronavirus was negative. An urgent computed tomography scan excluded significative colonic dilatation, but showed markedly thickened and enhanced colonic walls. A rectosigmoidoscopy showed severely inflamed mucosa with multiple deep ulcers; histology confirmed severely active ulcerative colitis. Broad-spectrum antibiotics, intravenous corticosteroids, and anti-thrombotic prophylaxis were started promptly. Despite the absence of urgent surgical indications, we thoroughly pondered the potential risk of performing urgent colectomy with post-surgical ICUs converted into critical COVID-19 units. We decided to transfer the patient to a COVIDfree hospital with IBD-specialized gastroenterologists and surgeons (Rho Hospital, in the Northern area of Milan) for further management. Ultimately, the patient responded only partially to intravenous steroids, with a dramatic fall in Creactive protein level $(0.95 \mathrm{mg} / \mathrm{dL})$, but persistent bloody diarrhea with up to 10 bowel movements. Salvage therapy with infliximab $5 \mathrm{mg} / \mathrm{kg}$ was then started with satisfying clinical efficacy, thus avoiding urgent colectomy.

This case clearly highlights some unanticipated difficulties in providing adequate care to patients with severe IBD in a highprevalence area of COVID-19. The limitation to all nonurgent consultations and the extreme pressure on the emergency system can lead to wide diagnostic and therapeutic delays. Moreover, many patients themselves may try to avoid access to hospitals, even in presence of severe symptoms because of the fear of getting infected. Severe IBD flares require admission, tight monitoring, and may require urgent surgery. All of these measures may become problematic during the pandemic. In our region, after the identification of the first COVID-19 clusters at the end of February, within a few days, several hospitals (included ours) were almost completely converted to COVID-19 clinics, with consequent deranging of physicians' organization chart and limitations of specialistic activities. As a third-level IBD center, we struggled to guarantee essential care to our patients, such as infusional therapies and urgent consultations, and to protect them from the risk of infection by instituting telephonic screening and 24/7 availability. ${ }^{3}$ However, with our gastroenterology ward closed and all gastroenterologists but 1 reassigned to new COVID units, we were unable to adequately manage patients with IBD flares.

Providing appropriate care to IBD patients during the COVID-19 pandemic may require a structural reorganization of IBD centers. If adequate counseling of patients, reallocation of limited resources, and creation of clean pathways are key measures to guarantee continuity of care to stable patients, the establishment of hospital networks with identification of dedicated hubs and the sensibilization of emergency care providers, general practitioners, and even general population are key measures in order to treat patients with severe IBD and to reduce the "collateral damages" of SARS-CoV-2.

\section{VINCENZO OCCHIPINTI}

Gastroenterology Unit

IRCCS Policlinico San Donato

San Donato Milanese, Italy

SIMONE SAIBENI

Gastroenterology Unit

Rho Hospital

ASST Rhodense

Lombardy, Italy

GIANLUCA M. SAMPIETRO

General Surgery Division

Rho Hospital

ASST Rhodense

Rho, Italy

LUCA PASTORELLI

Gastroenterology Unit

IRCCS Policlinico San Donato

San Donato Milanese, Italy and

Department of Biomedical Sciences for Health

University of Milan

Milan, Italy

\section{References}

1. Rubin DT, et al. Gastroenterology 2020;159:6-13.e6.

2. Livingston E, Bucher K. JAMA 2020;323:1335.

3. Occhipinti V, Pastorelli L. Inflamm Bowel Dis 2020; 26:793-796.

Conflicts of interest

This author discloses the following: S. Saibeni received lecture fees from Takeda Pharmaceuticals and Janssen Pharmaceuticals, member of Advisory Boards for AbbVie and Janssen Pharmaceuticals. The remaining authors disclose no conflicts.

Most current article

https://doi.org/10.1053/j.gastro.2020.05.027 